\title{
Trichostatin A induces p53-dependent endoplasmic reticulum stress in human colon cancer cells
}

\author{
LIMENG DAI $^{1}$, GANG HE $^{1}$, KUN ZHANG $^{2}$, XINGYING GUAN $^{1}$, YAN WANG $^{1}$ and BO ZHANG ${ }^{1}$ \\ Departments of ${ }^{1}$ Medical Genetics and ${ }^{2}$ Pathogenic Biology, College of Basic Medical Science, \\ Army Medical University (Third Military Medical University), Chongqing 400038, P.R. China
}

Received February 26, 2018; Accepted September 24, 2018

DOI: $10.3892 /$ ol.2018.9641

\begin{abstract}
Trichostatin A (TSA) has been demonstrated to exhibit various anticancer effects that influence cell cycle arrest, cell proliferation and apoptosis of cancer cells. A potential association between TSA and endoplasmic reticulum (ER) function has been suggested but its anticancer mechanism involving the induction of ER stress is unknown. p53 has previously been demonstrated to regulate ER function in response to stress but its role involving TSA and ER stress in cancer cells is poorly understood. The current study identified that TSA induced ER stress in wild type (WT) HCT116 human colon cancer cells. Following TSA treatment, the ER stress markers GRP78 and GRP94 significantly increased without hyperacetylation of their promoter regions. The inositol-requiring enzyme 1 $\alpha$ (IRE1 $\alpha$ )/X-box binding protein 1 (XBP1) pathway was implicated due to an association of phosphorylated IRE1 $\alpha$ and spliced XBP1 with ER stress. However, luciferase reporter assay indicated that splicing events were attenuated in HCT116 TP53(-/-) cells. Furthermore, cell viability and apoptosis were revealed to depend on p53 during TSA treatment. Cell viability increased and the apoptosis rate decreased in HCT116 TP53(-/-) cells compared with WT HCT116 cells undergoing TSA treatment. In conclusion, the current study revealed that TSA may induce ER stress via a p53-dependent mechanism in colon cancer cells. This provides information that may assist the development of treatments that exploit the anticancer function of TSA.
\end{abstract}

Correspondence to: Professor Bo Zhang, Department of Medical Genetics, College of Basic Medical Science, Army Medical University (Third Military Medical University), 30 Gaotanyan, Chongqing 400038, P.R. China

E-mail:bo_zhang@yahoo.com

Key words: trichostatin A, p53, endoplasmic reticulum stress, colon cancer

\section{Introduction}

Trichostatin A (TSA) is a histone deacetylase (HDAC) inhibitor of class I and II HDACs. TSA influences gene expression by interfering with the removal of acetyl groups from histones, thereby altering the balance between DNA transcription factors and chromatin (1). Based on the known medicinal properties of HDAC inhibitors, TSA has been tested as a treatment against various cancer cells with highly expressed HDACs, including colon (2), breast (3) and lung (4) cancer cells. TSA is known to affect the growth, apoptosis, autophagy and/or differentiation processes of these cancer cells. Previous studies have identified a potential association between TSA and endoplasmic reticulum (ER) function (5); however, to the best of our knowledge, an anticancer mechanism involving TSA and ER stress is unknown.

To investigate ER stress, dysfunction of the ER and the unfolded protein response was induced under adverse conditions, including metabolic and anaerobic stress, which disrupts the protein-folding function of the ER. Altered ER homeostasis results in an accumulation of unfolded or misfolded proteins, which ultimately leads to ER stress $(6,7)$. The ER stress response activates cytotoxic mechanisms involving a number of regulatory cytokines associated with the onset of programmed cell death, suggesting this is a possible target in the development of chemotherapeutic agents for inducing cancer cell toxicity (8). As an essential tumor suppressor, the TP53 gene regulates the processes of ER stress, apoptosis, DNA repair, cell cycle and nuclear vesicular trafficking, in the presence of cellular stressors, including hypoxia, DNA damage and oncogene activation $(9,10)$. Previous studies have revealed that p53 is upregulated in response to ER stress and participates in ER stress-induced apoptosis (11). However, to the best of our knowledge, the role of p53 in cancer cells exposed to TSA and ER stress is not understood.

In the current study, the anticancer effect of TSA on ER function was investigated in the HCT116 cell line. It was identified that ER stress was induced by TSA. Additionally, the inositol-requiring enzyme $1 \alpha$ (IRE1 $\alpha$ )/X-box binding protein 1 (XBP1) pathway was implicated in wild type (WT) HCT116 cells. Mutation or silencing of TP53 attenuated ER stress. Cell viability increased and the apoptosis rate decreased in HCT116 TP53(-/-) cells compared with WT HCT116 cells undergoing TSA treatment. Therefore, the induction 
of ER stress by TSA in colon cancer cells likely involves a p53-dependent mechanism.

\section{Materials and methods}

Materials. TSA and 4-phenylbutyrate were purchased from Merck KGaA (Darmstadt, Germany). Tunicamycin (TM; cat. no. 654380) were obtained from Sigma-Aldrich; Merck KGaA). Primary antibodies for GRP78 (cat. no. 3183), GRP94 (cat. no. 2104), p53 (cat. no. 2524) and IRE1 $\alpha$ (cat. no. 3294) were obtained from Cell Signaling Technology, Inc. (Danvers, MA, USA). Primary antibodies for XBP1 (cat. no. ab37151 and cat. no. ab220783) were purchased from Abcam (Cambridge, UK). A primary phosphospecific antibody for phosphorylated IRE1 $\alpha$ (p-IRE1 $\alpha$ ) was purchased from Abcam (cat. no. ab124945). Small interfering RNA (siRNA) of p53 was obtained from Shanghai GenePharma Co., Ltd (Shanghai, China). Transfection reagent Lipofectamine 2000 was obtained from Invitrogen (Thermo Fisher Scientific, Inc., Waltham, MA, USA). The Cell Counting kit-8 (CCK-8 kit) and the Annexin V-fluorescein isothiocyanate (FITC) Detection kit were obtained from Beyotime Institute of Biotechnology (Haimen, China).

Cell culture and TSA treatment. WT HCT116, HCT116 TP53(-/-) and HT29 human colon cancer cell lines were purchased from Shanghai Cell Bank (Shanghai, China; http://www.cellbank.org.cn). All three cell lines were cultured in Dulbecco's modified Eagle's medium (Hyclone, Logan, UT, USA) with $10 \%$ fetal bovine serum (Gibco; Thermo Fisher Scientific, Inc.) at $37^{\circ} \mathrm{C}$ in a humidified incubator containing $5 \% \mathrm{CO}_{2}$. For the TSA treatment, specific amounts of TSA were added to the medium to generate a mixed medium gradient with different concentrations of TSA $(0,0.04,0.2$ and $1 \mu \mathrm{M})$.

Cell viability. Cells were seeded in 96-well plates with $5 \times 10^{3}$ cells per well. Following $24 \mathrm{~h}$ of cultivation, the negative control siRNAs were transfected with Lipofectamine 2000 according to the manufacturer's protocol. Next, the cells were treated with different concentrations of TSA $(0,0.04,0.2$ and $1 \mu \mathrm{M}$ ) and cultured at $37^{\circ} \mathrm{C}$ for $24 \mathrm{~h}$. Cell viability was determined using the CCK-8 assay according to the manufacturer's protocol, with a $2 \mathrm{~h}$ incubation. The number of viable cells was quantified by measuring the absorbance at $450 \mathrm{~nm}$ using a microplate reader. Three replicates were performed for each group.

Apoptosis analysis. Apoptosis was analyzed using the Annexin V-FITC Apoptosis Detection kit according to the manufacturer's protocol. Trypsin was used to collect $1 \times 10^{6}$ cells with or without TSA treatment at a concentration of $1 \mu \mathrm{M}$ for $24 \mathrm{~h}$. These collected cells were washed twice with cold PBS and resuspended with Annexin V-FITC binding solution. Propidium iodide was used to stain the cells for $20 \mathrm{~min}$ at $25^{\circ} \mathrm{C}$. Cells were detected using a FACSCalibur flow cytometer and analyzed by BD CellQuest Pro 5.1 software (both BD Biosciences, Franklin Lakes, NJ, USA).

Reporter assay. Luciferase reporter pCAX-F-XBP1delDBDLuc (provided by Dr Takao Iwawaki, Gunma University, Japan) was used to detect XBP1 splicing events (12). The luciferase is expressed only when XBP1 splicing occurs and its activity reflects the number of splicing events. TM treatment was used as positive control. The vectors were transfected using Lipofectamine 2000 (Invitrogen; Thermo Fisher Scientific, Inc.). Cell lysate with or without drug treatment was collected $24 \mathrm{~h}$ following transfection. Luciferase activity was evaluated using the Luciferase Assay system (Promega Corporation, Madison, WI, USA) according to the manufacturer's protocol.

RNA extraction and reverse transcription-quantitative polymerase chain reaction $(R T-q P C R)$. Total RNA from the cultured cells was extracted using RNAiso Plus reagent (Takara Bio, Inc., Otsu, Japan) and quantified with a NanoDrop spectrophotometer (Thermo Fisher Scientific, Inc.). The purified RNA $(0.5 \mu \mathrm{g})$ was reverse transcribed to cDNA using PrimeScript RT reagent kit (Takara Biotechnology Co., Ltd., Dalian, China), according to the manufacturer's protocols. Then qPCR was performed using a Bio-Rad CFX Connect detection system (Bio-Rad Laboratories, Inc., Hercules, CA, USA) with SYBR Premix Ex Taq II (Takara Bio, Inc.; cat. no. RR820L). A two-step PCR protocol was used with the following conditions: $95^{\circ} \mathrm{C}$ for $30 \mathrm{sec}$, followed by 40 cycles of $95^{\circ} \mathrm{C}$ for $5 \mathrm{sec}$ and $60^{\circ} \mathrm{C}$ for $30 \mathrm{sec}$. All reactions were carried out in triplicate. The mRNA expression levels of target genes were normalized to the mRNA expression of GAPDH using the $2^{-\Delta \Delta C q}$ method (13). The sequences of the forward and reverse primers are presented in Table I.

Western blot analysis. Cells were suspended in ice-cold radioimmunoprecipitation assay buffer, which contained protease inhibitors (Beyotime Institute of Biotechnology). The extraction mixture was collected and centrifuged at 10,000 x g at $4^{\circ} \mathrm{C}$ for $15 \mathrm{~min}$. The supernatant was obtained and the protein concentration was analyzed using a Bradford assay. Equal amounts of protein extractions $(40 \mu \mathrm{g})$ were separated on $10 \%$ SDS-polyacrylamide gels and electrophoretically transferred onto polyvinylidene difluoride membranes (EMD Millipore, Billerica, MA, USA). Subsequent to being blocked with $5 \%$ skimmed milk for $2 \mathrm{~h}$ at room temperature, membranes were incubated with a primary antibody at $4^{\circ} \mathrm{C}$ overnight. The following antibodies were used for western blotting: Anti-GRP78 (dilution, 1:1,000), anti-GRP94 (dilution, 1:1,000), anti-p53 (dilution, 1:1,000), anti-IRE1 $\alpha$ (dilution, 1:1,000), anti-p-IRE1 $\alpha$ (dilution, 1:1,000), anti-XBP1-U (dilution,1:1,000; cat. no. ab37151), anti-XBP1-S (dilution, 1:1,000; cat. no. ab220783) and anti-GAPDH (dilution, 1:5,000). The blots were then incubated with anti-mouse (cat. no. zb2305) or anti-rabbit (cat. no. zb2301) secondary antibodies at a dilution of 1:5,000) coupled to horseradish peroxidase (Zhongshan Golden Bridge Biotechnology, Beijing, China) at $4^{\circ} \mathrm{C}$ for $2 \mathrm{~h}$. The immunoreactive proteins were detected using the ECL Detection system (Thermo Fisher Scientific, Inc.). Densitometric analysis of the protein bands was conducted using Image J 1.42 software (National Institutes of Health, Bethesda, MD, USA). Analysis of each sample was repeated three times.

Chromatinimmunoprecipitation (ChIP)andsemi-quantitative PCR. A total of $1 \times 10^{7}$ cells, with or without $1 \mu \mathrm{M}$ TSA 
Table I. Primers for reverse transcription-quantitative PCR and semi-quantitative PCR.

\begin{tabular}{|c|c|c|}
\hline Target & Target sequence & Products, bp \\
\hline GRP78 mRNA & $\begin{array}{l}\text { F: CGTCCTATGTCGCCTTCACT } \\
\text { R: TGTCTTTGTTTGCCCACCTC }\end{array}$ & 230 \\
\hline GRP94 mRNA & $\begin{array}{l}\text { F: CAGTTTGGTGTCGGTTTCTA } \\
\text { R: AGTGTTTCCTCTTGGGTCAG }\end{array}$ & 141 \\
\hline GAPDH mRNA & $\begin{array}{l}\text { F: GGGAAGGTGAAGGTCGGAGTC } \\
\text { R: CCTGGAAGATGGTGATGGGAT }\end{array}$ & 232 \\
\hline XBP1 mRNA & $\begin{array}{l}\text { F: GAACCAGGAGTTAAGACAGCG } \\
\text { R: CCAGAATGCCCAACAGGATA }\end{array}$ & 212 \\
\hline p21 promoter & $\begin{array}{l}\text { F: GAGGGACTGGGGGAGGAGGGAA } \\
\text { R: CCACAAGGAACTGACTTCGGCA }\end{array}$ & 267 \\
\hline GRP78 promoter & $\begin{array}{l}\text { F: GAAATTGCGCTGTGCTCCTGTG } \\
\text { R: CGTCCTCCTTCTTGTCCTCCTCC }\end{array}$ & 149 \\
\hline GRP94 promoter & $\begin{array}{l}\text { F: CCTCACGAATCCTCATTGGGT } \\
\text { R: TCGGATCCTCACACCTCCAGC }\end{array}$ & 158 \\
\hline GAPDH promoter & $\begin{array}{l}\text { F: CGGCTCCAATTCCCCATCTC } \\
\text { R: GAGGTGATCGGTGCTGGTTC }\end{array}$ & 132 \\
\hline
\end{tabular}

F, forward; R, reverse; XBP1, X-box binding protein 1; bp, base pair; PCR, polymerase chain reaction.

treatment at $37^{\circ} \mathrm{C}$ with $5 \% \mathrm{CO}_{2}$ for $24 \mathrm{~h}$, were harvested and washed with cold PBS. A ChIP assay was performed using an EZ-ChIP kit from EMD Millipore according to the manufacturer's protocol. The cross-linked chromatins were sonicated $(300 \mathrm{~W}$, Pulse $1 \mathrm{~s} / 3 \mathrm{~s})$ for $2 \mathrm{~min}$ at $0^{\circ} \mathrm{C}$ and immunoprecipitated overnight using acetylated histone $\mathrm{H} 3$ antibody (dilution, 1:300; cat. no. 17-615) or anti-IgG (dilution, 1:1,000; cat. no. 17-371; both Upstate Biotechnology, Inc., Lake Placid, NY, USA) antibody. The precipitated DNA was detected by semi-quantitative PCR using GoTaq ${ }^{\circledR}$ Master Mix (Promega Corporation, Madison, WI, USA), according to the manufacturer's protocols, using the following conditions: $95^{\circ} \mathrm{C}$ for $30 \mathrm{sec}$, followed by 15 cycles of $95^{\circ} \mathrm{C}$ for $5 \mathrm{sec}, 60^{\circ} \mathrm{C}$ for $30 \mathrm{sec}$ and $72^{\circ} \mathrm{C}$ for $30 \mathrm{sec}$. A total of $5 \mu \mathrm{l}$ PCR products were stained with $1 \mu \mathrm{l}$ 4S Green Plus (Sangon Biotech Co. Ltd., Shanghai, China) for $10 \mathrm{sec}$ at room temperature, according to the manufacturer's protocols, and detected by $5 \%$ agarose gel electrophoresis For histone hyperacetylation analysis, the p21 promoter was used as a positive control (14) and the GAPDH promoter was used as a reference region. Quantitative values obtained for the PCR products (GRP78, GRP94 and p21) were normalized relative to the GAPDH promoter and adjusted by the amount of normal control group. Analysis of the bands of PCR products was conducted using ImageJ 1.42 software (National Institutes of Health, Bethesda, MD, USA). Analysis of each sample was repeated three times. Relevant primers are listed in Table I.

Knockdown of TP53 expression by siRNA. Cells were transfected with TP53 siRNA, while the control was transfected with negative control siRNA (GenePharma Co., Ltd) using Lipofectamine 2000, according to the manufacturer's protocol. Cells were harvested $24 \mathrm{~h}$ post-transfection and used for
Table II. Sequences of siRNA against TP53 and NC.

\begin{tabular}{lc}
\hline Name & \multicolumn{1}{c}{ Target sequence } \\
\hline TP53 siRNA & 5'-CUACUUCCUGAAAACAACGTT-3' \\
& 5'-CGUUGUUUUCAGGAAGUAGTT-3' \\
NC siRNA & 5'-UUCUCCGAACGUGUCACGUTT-3' \\
& 5'-ACGUGACACGUUCGGAGAATT-3' \\
\hline
\end{tabular}

NC, negative control; siRNA, small interfering RNA.

subsequent assays. The interference effect of p53 was assessed by western blotting. The siRNA sequences are presented in Table II.

Statistical analysis. All data were presented as the mean \pm standard error and each experiment was performed independently at least three times. Student's t-test and analysis of variance (ANOVA) were used for detecting differences between experimental groups. Multiple group comparisons were carried out by ANOVA with a post hoc Student-Newman-Keuls test. $\mathrm{P}<0.05$ was considered to indicate a statistically significant difference. Statistical analysis was carried out using SPSS 16.0 software (SPSS Inc., Chicago, IL, USA).

\section{Results}

TSA induces p53-dependent ER stress. To determine the effect of TSA on ER stress, HCT116 cells were treated with TSA at increasing concentrations for specific time intervals, after which the expression of GRP78 and GRP94 was 

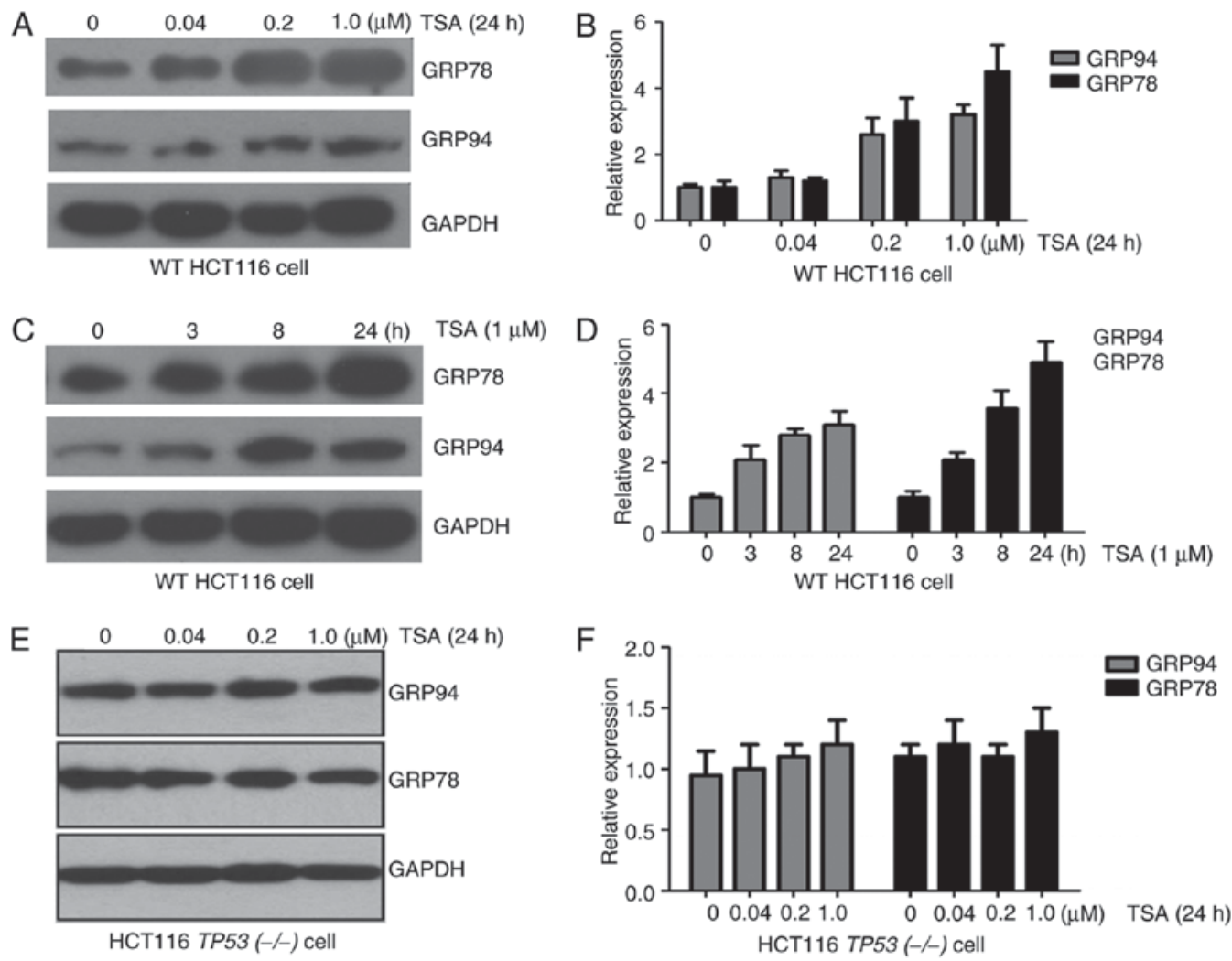

Figure 1. GRP78 and GRP94 expression is associated with p53 expression in human colon cancer cells. The expression levels of GRP78 and GRP94 (A) proteins and (B) mRNA in WT HCT116 cells increased following TSA treatment in a dose-dependent manner. Significant statistical differences were indicated for GRP78 and GRP94 at different concentration of TSA ( 1 vs. $0.2 \mu \mathrm{M} ; 0.2$ vs. $0.04 \mu \mathrm{M} ; 0.2$ vs. $0 \mu \mathrm{M}$ ), respectively; P<0.01). The expression levels of GRP78 and GRP94 (C) proteins and (D) mRNA in WT HCT116 cells increased following treatment with $1 \mu \mathrm{M}$ TSA in a time-dependent manner. Significant statistical differences were indicated for GRP78 and GRP94 between different time points groups $(0,3,8$ and 24; 0 P<0.01). No association was identified between TSA treatment and the expression levels of GRP78 and GRP94 (E) proteins and (F) mRNA in HCT116 TP53(-/-) cells. TSA, trichostatin A; WT, wild type.

analyzed. As demonstrated in Fig. 1A-D, the expression of ER stress markers GRP78 and GRP94 increased at the mRNA and protein level, in a dose- and time-dependent manner with TSA treatment.

Since HCT116 cells contained the WT TP53 gene, the response to TP53 was investigated in another colon cancer cell line, HT29, in which the TP53 gene was mutated. Increased expression of GRP78 and GRP94 was not observed in HT29 cells treated with TSA compared with untreated cells (data not presented). In addition, HCT116 TP53(-/-) cells were treated with TSA. As observed in HT29 cells, expression of GRP78 and GRP94 in HCT116 TP53(-/-) cells was not associated with TSA treatment (Fig. 1E and F). These results suggested that TSA-induced expression of ER stress marker proteins depends on $\mathrm{p} 53$.

Hyperacetylation of ER stress marker genes is not induced by TSA. TSA, a classic HDAC inhibitor, disrupts the balance between histone acetylation, deacetylation and induced hyperacetylation, which alters chromatin structure and gene expression (1). Since TSA was identified to increase gene expression of ER stress markers at the transcriptional level (Fig. 1B and D), the current study investigated if this increased gene expression was associated with hyperacetylation of ER stress markers. Following TSA treatment, the acetylation of histone H3K9 in the promoter regions of GRP78 and GRP94 genes was analyzed. ChIP and a semi-quantitative PCR assay were performed to measure the acetylation level of histone H3K9 in the GRP78 and GRP94 promoter regions. As demonstrated in Fig. 2, no significant differences were identified in the acetylation levels of the promoter regions of GRP78, GRP94 and GAPDH in TSA-treated cells. However, hyperacetylation was significantly increased in the positive control p21 gene promoter region following TSA treatment. Therefore, other mechanisms likely contribute to the increase of ER stress-marker expression following TSA treatment.

p53 is associated with the viability and apoptosis of HCT116 cells following TSA treatment. To assess the association of p53 with the cytotoxicity of TSA, cell viability and apoptosis assays were performed with WT HCT116 and HCT116 TP53(-/-) cells. A CCK-8 assay revealed that an increase in the concentration of TSA was associated with a decrease in the viability of WT HCT116 and HCT116 TP53(-/-) cells. A significant difference was identified in the viability of WT HCT116 and HCT116 TP53(-/-) cells treated with $1 \mu \mathrm{M}$ TSA (Fig. 3A). Additionally, the apoptosis rate of WT HCT116 cells was identified to be significantly higher compared with the apoptosis rate of HCT116 TP53(-/-) cells following treatment with $1 \mu \mathrm{M}$ TSA (Fig. 3B and C). These results suggested that p53 expression was associated with the cell viability and apoptosis rate of colon cancer cells treated with $1 \mu \mathrm{M}$ TSA. 

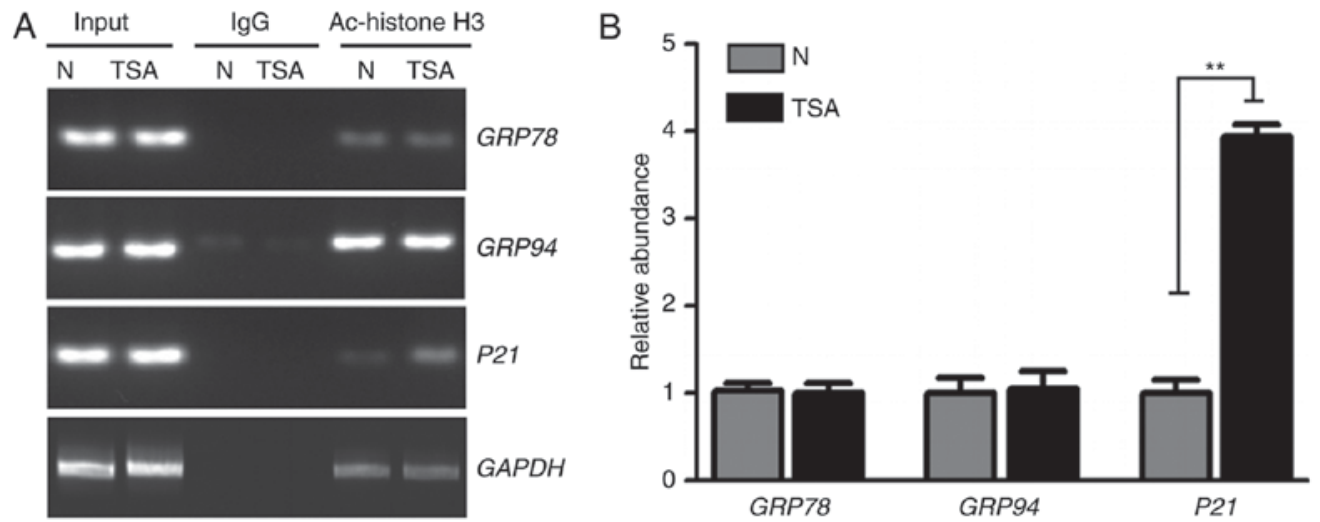

Figure 2. Hyperacetylation of GRP78 and GRP94 promoter regions is not induced by TSA. (A) Semi-quantitative PCR of WT HCT116 cells using H3K9Ac antibody or IgG. PCR production of GRP78, GRP94 and GAPDH promoter regions was not altered but the positive control p21 promoter region increased following TSA treatment. (B) Quantification results for PCR production of p21, GRP78 and GRP94 promoter regions using H3K9Ac antibody. Quantitative values obtained were normalized relative to the GAPDH promoter. ${ }^{* *} \mathrm{P}<0.01$. TSA, trichostatin A; WT, wild type; PCR, polymerase chain reaction; N, negative control; IgG, immunoglobulin G; Ac, acetylation.

A
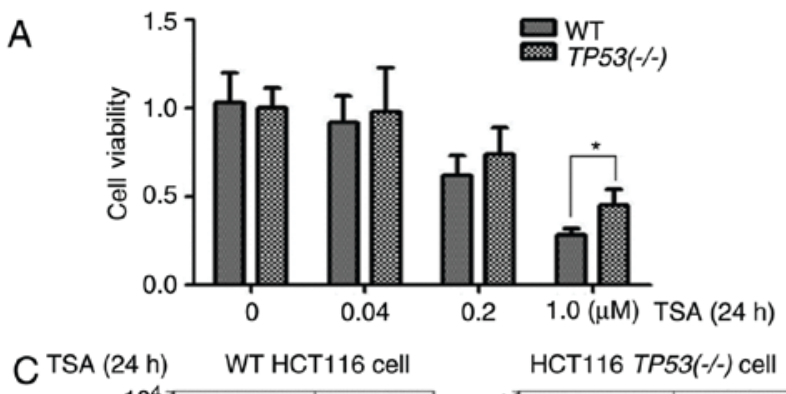

HCT116 TP53(-/) cell
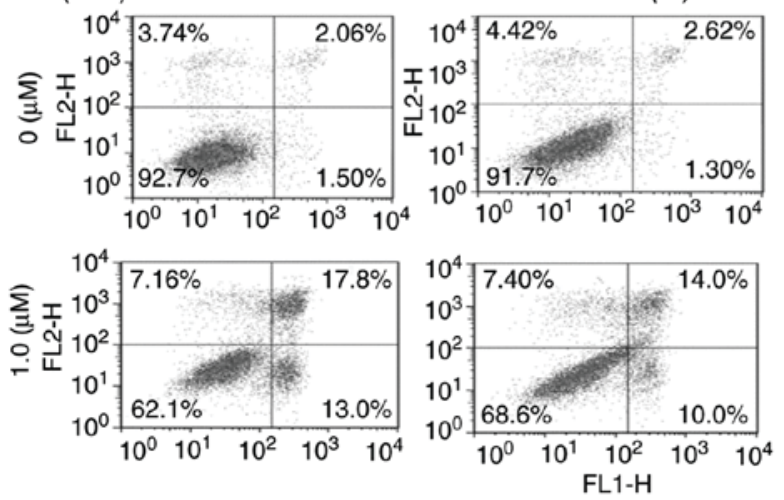

E

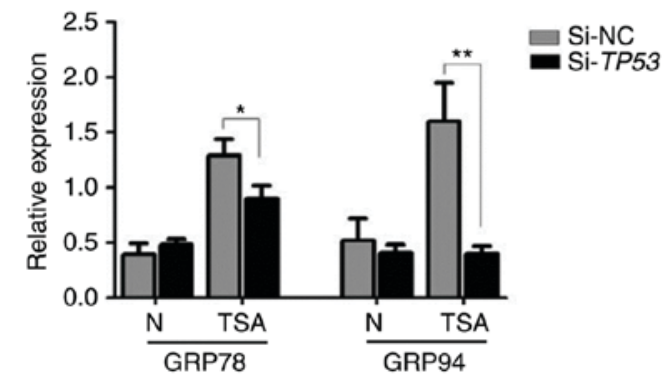

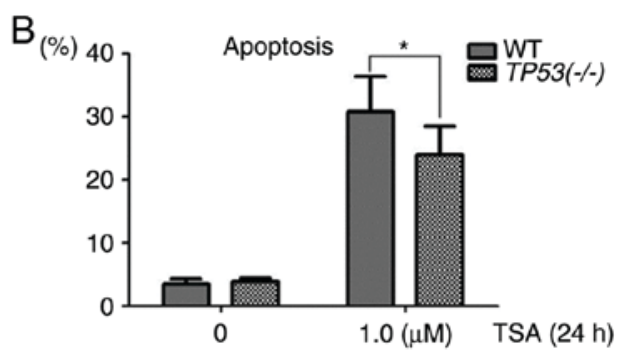

D
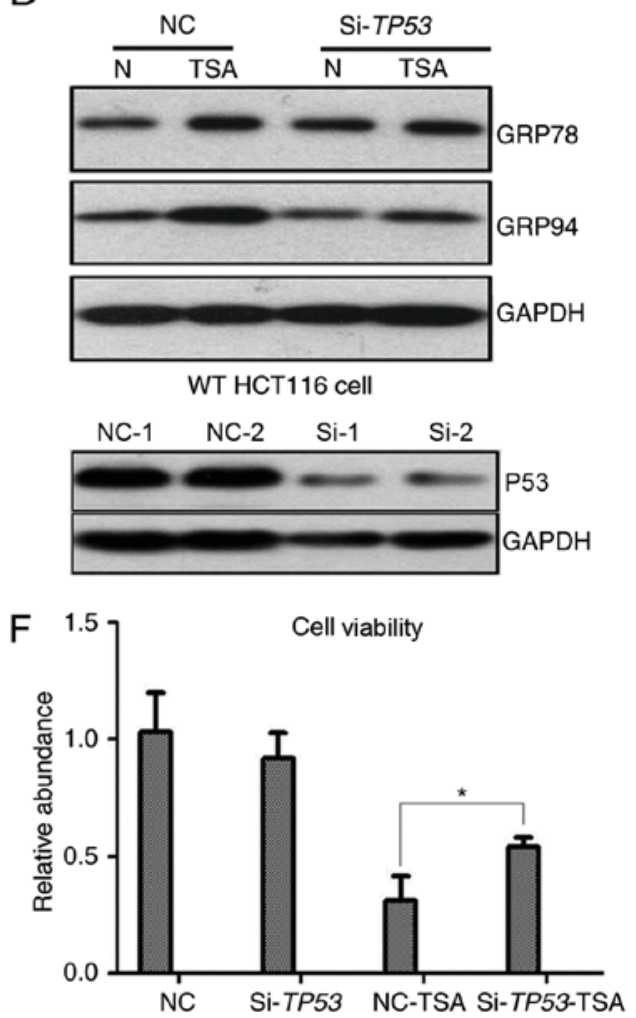

Figure 3. Cell viability and apoptosis analyses for the role of p53 in TSA treatment. (A) Cell viability decreased with increasing concentration of TSA in WT HCT116 and HCT116 TP53(-/-) cells. Following treatment with $1 \mu \mathrm{M}$ TSA for $24 \mathrm{~h}$, the cell viability was significantly decreased in WT HCT116 cells compared with HCT116 TP53(-/-) cells. (B and C) The apoptosis rate of WT HCT116 and HCT116 TP53(-//) cells increased following treatment with $1 \mu$ M TSA for $24 \mathrm{~h}$. The apoptosis rate was significantly higher in WT HCT116 cells compared with HCT116 TP53(-/-) cells. (D and E) siRNAs could efficiently inhibit p53 expression compared with NCs in HCT116 cells. Western blot analysis indicated that silencing of TP53 decreased the expression of GRP78 and GRP94 in WT HCT116 cells. (F) Silencing of TP53 significantly increased the cell viability of WT HCT116 cells compared with NC cells following treatment with $1 \mu \mathrm{M}$ TSA for $24 \mathrm{~h} .{ }^{*} \mathrm{P}<0.05,{ }^{* *} \mathrm{P}<0.01$. TSA, trichostatin A; WT, wild type; siRNA, small interference RNA; NC, negative control; Si, small interference; $\mathrm{N}$, no treatment. 

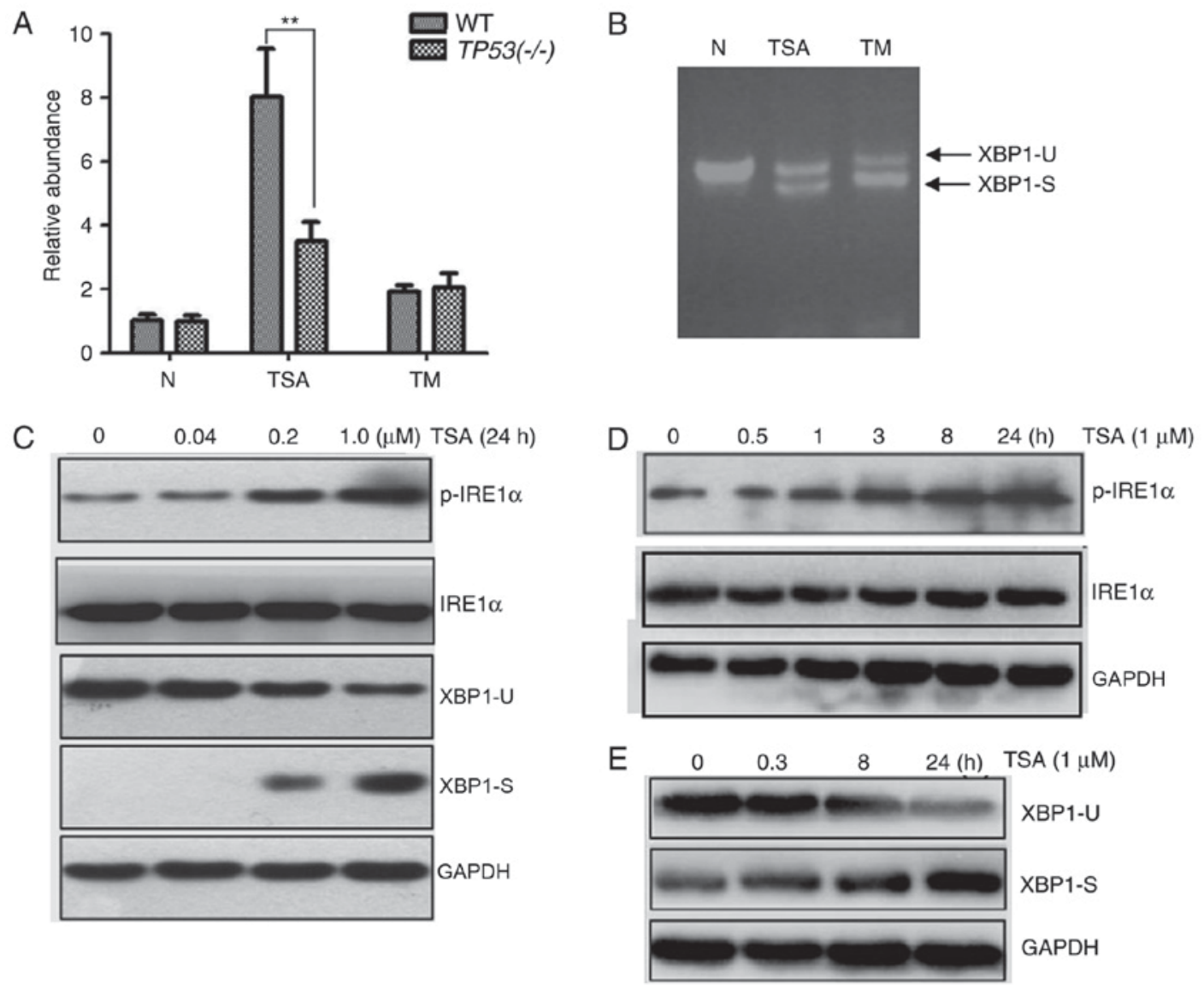

Figure 4. Effect of TSA treatment on the IRE1 $/$ XBP1 pathway. In the luciferase reporter assay, cells were transfected with pCAX-F-XBP1delDBD-Luc. (A) An increase in luciferase activity was associated with TSA and TM treatment at a concentration of $1 \mu \mathrm{M}$ for $24 \mathrm{~h}$. When treated with TSA the luciferase activity was significantly higher in WT HCT116 cells compared with HCT116 TP53(-/-) cells. (B) The spliced form of XBP1 was detected by reverse transcription-quantitative polymerase chain reaction. (C) A decrease of XBP1-U and an increase of XBP1-S were associated with an increasing concentration of TSA. Similarly, an increase of p-IRE1 $\alpha$ was associated with an increasing concentration of TSA. (D) An increase of p-IRE1 $\alpha$ was associated with the treatment time of $1 \mu \mathrm{M}$ TSA. (E) An increase of XBP1-S and a decrease of XBP1-U was associated with the treatment time of $1 \mu \mathrm{M}$ TSA. ${ }^{* *} \mathrm{P}<0.01$. TSA, trichostatin A; IRE1 $\alpha$, inositol-requiring enzyme $1 \alpha$; XBP1, X-box binding protein 1; TM, tunicamycin; WT, wild type; XBP1-U, unspliced XBP1; XBP1-S, spliced XBP1; p-IRE1 $\alpha$, phosphorylated IRE1 $\alpha$.

Silencing of p53 attenuates ER stress in WT HCT116 cells. ER stress induced by TSA was detected in WT HCT116 cells but not in HCT116 TP53(-/-) cells. To further investigate the role of p53 in TSA treatment, TP53 was knocked down by RNA interference in WT HCT116 cells. As expected, siRNA efficiently inhibited p53 expression in WT HCT116 cells (Fig. 3D). The molecular response following treatment with TSA was detected by immunoblotting; following TSA treatment the protein levels of GRP78 and GRP94 were significantly higher in WT HCT116 cells compared with HCT116 cells in which TP53-knockdown had been achieved by siRNA (Fig. 3D and E). A cell viability assay demonstrated that in comparison with the negative control group, cell viability was significantly higher in TP53-knockdown WT HCT116 cells following TSA treatment (Fig. 3F).

Involvement of the IRE1 pathway with TSA treatment. A previous study has suggested that the IRE1 pathway is involved in the ER stress response (15). The RNase activity of IRE1 $\alpha$ is activated following ER stress and its spliced form may reflect the activation of IRE1 $\alpha$ (16).

To identify whether the IRE1 pathway is associated with TSA-induced ER stress, a luciferase reporter
pCAX-F-XBP1delDBD-Luc was used to detect XBP1 splicing events. The luciferase was only expressed when XBP1 splicing occurred and its expression level was associated with the number of splicing events. As demonstrated in Fig. 4A, an increase in luciferase activity was associated with TSA treatment. The increase in luciferase activity was markedly higher in TSA-treated WT HCT116 cells compared with the positive control WT HCT116 cells treated with $1 \mu \mathrm{M}$ TM for $24 \mathrm{~h}$. However, this increase in luciferase activity was significantly smaller in TSA-treated HCT116 TP53(-/-) cells.

Next, the spliced form of XBP1 was detected by semi-quantitative PCR. As demonstrated in Fig. 4B, unspliced XBP1 and spliced XBP1 were detected in TSA- and TM-treated WT HCT116 cells. Additionally, the protein levels of p-IRE1 $\alpha$ were measured. As expected, the protein level of unspliced XBP1 decreased and spliced XBP1 increased following TSA treatment, in a dose-dependent manner (Fig. 4C). Similarly, the protein levels of p-IRE1 $\alpha$ increased following TSA treatment, in a dose-dependent manner (Fig. 4C). Furthermore, levels of p-IRE1 $\alpha$ and spliced XBP1 increased in a time-dependent manner with TSA treatment (Fig. 4D and E). Taken together, these data suggested that the IRE1 pathway may be involved in TSA-induced ER stress. 


\section{Discussion}

In the current study, the expression of ER stress markers, GRP78 and GRP94, was revealed to be increased following TSA treatment of WT HCT116. Furthermore, this TSA-induced ER stress was identified to be associated with the expression of p53. Previously, a study identified that HDAC inhibitors specifically induce the expression of GRP78 and this effect is amplified by ER stress (17). In the current study, GRP78 and GRP94 were upregulated in a p53-dependent manner. However, GRP78 and GRP94 promoter regions were not hyperacetylated following TSA treatment. Previous studies have identified that there are HDAC1 response elements in the promoter region of GRP78 that function as negative regulation factors $(17,18)$. This suggests that TSA may inhibit HDAC1 and induce GRP78 expression. In addition, TSA may activate the gene promoter through the Sp1 and Sp3 sites, including ethanolamine kinase 1 gene (19). These results suggest that the increase in ER stress markers may involve transcription factors or regulatory elements, including HDAC1 response elements, Sp1 and Sp3 sites, and factors within the p53 network. However, these suggestions require verification in future studies.

To further investigate the effect of increased ER stress markers following TSA treatment, the associated pathways were analyzed. Previous studies have identified that the ER transmembrane proteins, IRE1 $\alpha$, protein kinase R-like ER kinase (PERK), eukaryotic initiation factor 2 (eIF2) and activating transcription factor 6 (ATF6), mediate the unfolded protein response and ER stress response in mammalian cells (20). ER stress, a highly conserved cellular defense mechanism, responds to perturbations of ER function (20). When ER stress occurs, the GRP78 and GRP94 proteins dissociate from ER membrane receptors, followed by activation of the unfolded protein response. Previous studies have revealed that the IRE1 $\alpha / \mathrm{XBP} 1$ pathway is important for oncogenesis, as it helps tumor cells adapt to ER stress and associated growth factors (21). In the current study, the IRE1 $\alpha / \mathrm{XBP} 1$ pathway was identified to be associated with TSA treatment. Levels of p-IRE1 $\alpha$ and spliced XBP1 increased following TSA-induced ER stress in cancer cells. However, no association was identified between TSA and ER stress when p53 was silenced. Silencing of p53 in WT HCT116 cells attenuated ER stress marker expression induced by TSA and decreased XBP1 splicing events. A previous study identified p53 as a crucial regulator of ER function; loss of p53 function induced an upregulation of IRE1 $\alpha$ expression, which increased ER function. In p53 deficient cells, the IRE1 $\alpha /$ XBP1 pathway was upregulated but the ER stress-dependent activation of ATF6 and the PERK/eIF2 $\alpha$ pathway was suppressed (22). In the current study, TSA induced ER stress in a p53-dependent manner; the expression of ER stress markers did not increase following TSA treatment when p53 function was lost. These data indicate that activation of the IRE1 $\alpha / \mathrm{XBP} 1$ pathway is associated with the upregulation of ER stress markers following TSA treatment. However, other pathways associated with ER stress were not analyzed in the current study. Therefore, the results primarily revealed the association between TSA and the IRE1 $\alpha / \mathrm{XBP} 1$ pathway.
TSA-induced apoptosis is an important function for cancer therapy; 553 has been identified to influence the antitumor effect of TSA (4). Tumor cells experience constant ER stress and are exposed to various stress stimuli in their microenvironment, including the accumulation of misfolded proteins, hypoxia, acidic $\mathrm{pH}$ levels, reactive oxygen species, calcium imbalance, viral proteins and hypoglycemia (23). Therefore, substantial ER stress may easily be induced in tumor cells, which may lead to tumor cell death. There are three pathways involved in ER stress mediated apoptosis: The C/EBP homologous protein (CHOP) associated pathway, the tumor necrosis factor receptor-associated factor-2/c-Jun N-terminal kinase (JNK) pathway and caspase-12 activation (24). The current study revealed that the IRE1 $\alpha / \mathrm{XBP} 1$ pathway may initiate downstream reactions. IRE1 $\alpha$ activation can induce JNK phosphorylation and phosphorylated JNK can exert proapoptotic effects by activating caspase-12/4 and caspase-8 (7). Additionally, XBP1 is known to activate the transcription of CHOP (25), therefore XBP1 contributes to the induction of apoptosis.

A mutation in the TP53 gene is often identified in human cancer cells (26). Activation of p53 has been revealed to suppress the activity of the mechanistic target of rapamycin and inhibit the translation of certain proteins (26). The elimination of p53 may provide an opportunity for cancer cells to promote unregulated proliferation due to increased protein synthesis and a subsequent increase in ER function (9). In the current study, the expression of p53 affected the extent of TSA-induced ER stress. p53 was associated with cell viability and apoptosis following TSA treatment; the rate of apoptosis was significantly higher in WT HCT116 cells compared with HCT116 TP53(-/-) cells following TSA treatment. A previous study has identified that ER stress may activate proapoptotic p53-target genes, including NOXA and p53 upregulated modulator of apoptosis; these genes are associated with the induction of cell death during ER stress (27). Therefore, p53 inactivation may be a driving factor for chemoresistance to TSA in HCT116 cells. Furthermore, a previous study has reported a combined effect of TSA with other factors, including iron chelators (18). Iron chelators promote binding between HDAC-1 and the GRP78 promoter region, which reduces GRP78 expression and promotes the chemotherapeutic effect of TSA. Knockdown of GRP78 also promotes desensitization to TSA in cancer cells, which reduces apoptosis (17). The observation made by the current study that the status of p53 influences the association between ER stress and TSA may provide insight into the development of an anticancer function of TSA.

In summary, the current study has identified that ER stress is induced by TSA and the IRE1 $\alpha / \mathrm{XBP} 1$ pathway in WT HCT116 cells. Mutation or silencing of TP53 has been revealed to attenuate ER stress. Furthermore, the current study identified that cell viability increased and the apoptosis rate decreased in HCT116 TP53(-/-) cells compared with WT HCT116 cells following TSA treatment. Overall the data reveal that TSA-induced ER stress may occur via a p53-dependent mechanism in colon cancer cells.

\section{Acknowledgements}

Not applicable. 


\section{Funding}

The current study was supported by the National Natural Science Foundation of China (grant nos. 31671331 and 81700222) and the Natural Science Foundation Project of CQ CSTC (grant no. cstc2017jcyjAX0442).

\section{Availability of data and materials}

All data generated or analyzed during the present study are included in this published article.

\section{Authors' contributions}

BZ and LD conceived the study. BZ and LD designed the study. LD, GH, KZ and XG performed the experiment. LD and $\mathrm{YW}$ analyzed the data. LD and $\mathrm{BZ}$ wrote the manuscript.

\section{Ethics approval and consent to participate}

Not applicable.

\section{Patient consent for publication}

Not applicable.

\section{Competing interests}

The authors declare that they have no competing interests.

\section{References}

1. Vanhaecke T, Papeleu P, Elaut G and Rogiers V: Trichostatin A-like hydroxamate histone deacetylase inhibitors as therapeutic agents: Toxicological point of view. Curr Med Chem 11: 1629-1643, 2004.

2. Liu Y, He G, Wang Y, Guan X, Pang X and Zhang B: MCM-2 is a therapeutic target of Trichostatin A in colon cancer cells. Toxicol Lett 221: 23-30, 2013.

3. Liu J and Li Y: Trichostatin A and Tamoxifen inhibit breast cancer cell growth by miR-204 and ER $\alpha$ reducing AKT/mTOR pathway. Biochem Biophys Res Commun 467: 242-247, 2015.

4. Wu TC, Lin YC, Chen HL, Huang PR, Liu SY and Yeh SL: The enhancing effect of genistein on apoptosis induced by trichostatin A in lung cancer cells with wild type p53 genes is associated with upregulation of histone acetyltransferase. Toxicol Appl Pharmacol 292: 94-102, 2016.

5. Yu L, Lu M, Wang P and Chen X: Trichostatin A ameliorates myocardial ischemia/reperfusion injury through inhibition of endoplasmic reticulum stress-induced apoptosis. Arch Med Res 43: 190-196, 2012.

6. Ron D and Walter P: Signal integration in the endoplasmic reticulum unfolded protein response. Nat Rev Mol Cell Biol 8: 519-529, 2007.

7. Montalbano R, Waldegger P, Quint K, Jabari S, Neureiter D, Illig R, Ocker M and Di Fazio P: Endoplasmic reticulum stress plays a pivotal role in cell death mediated by the pan-deacetylase inhibitor panobinostat in human hepatocellular cancer cells. Transl Oncol 6: 143-157, 2013.
8. Johnson GG, White MC and Grimaldi M: Stressed to death: Targeting endoplasmic reticulum stress response induced apoptosis in gliomas. Curr Pharm Des 17: 284-292, 2011.

9. Byun S, Namba T and Lee SW: Losing p53 loosens up ER-stress. Aging (Albany NY) 7: 895-896, 2015.

10. Jiang P, Du W, Mancuso A, Wellen KE and Yang X: Reciprocal regulation of $\mathrm{p} 53$ and malic enzymes modulates metabolism and senescence. Nature 493: 689-693, 2013.

11. Jeong K, Kim SJ, Oh Y, Kim H, Lee YS, Kwon BS, Park S, Park KC, Yoon KS, Kim SS, et al: p53 negatively regulates Pin1 expression under ER stress. Biochem Biophys Res Commun 454: 518-523, 2014.

12. Iwawaki T and Akai R: Analysis of the XBP1 splicing mechanism using endoplasmic reticulum stress-indicators. Biochem Biophys Res Commun 350: 709-715, 2006.

13. Livak KJ and Schmittgen TD: Analysis of relative gene expression data using real-time quantitative PCR and the 2(-Delta Delta C(T)) method. Methods 25: 402-408, 2001.

14. Sowa Y, Orita T, Hiranabe-Minamikawa S, Nakano K, Mizuno T, Nomura $\mathrm{H}$ and Sakai T: Histone deacetylase inhibitor activates the p21/WAF1/Cip1 gene promoter through the Sp1 sites. Ann N Y Acad Sci 886: 195-199, 1999.

15. Yoshida H, Matsui T, Yamamoto A, Okada T and Mori K: XBP1 mRNA is induced by ATF6 and spliced by IRE1 in response to ER stress to produce a highly active transcription factor. Cell 107: 881-891, 2001.

16. Kennedy D, Samali A and Jäger R: Methods for studying ER stress and UPR markers in human cells. Methods Mol Biol 1292: 3-18, 2015.

17. Baumeister P, Dong D, Fu Y and Lee AS: Transcriptional induction of GRP78/BiP by histone deacetylase inhibitors and resistance to histone deacetylase inhibitor-induced apoptosis. Mol Cancer Ther 8: 1086-1094, 2009.

18. Kilinc V, Bedir A, Okuyucu A, Salis O, Alacam H and Gulten S: Do iron chelators increase the antiproliferative effect of trichostatin A through a glucose-regulated protein 78 mediated mechanism? Tumour Biol 35: 5945-5951, 2014.

19. Kuan CS, See Too WC and Few LL: Sp1 and Sp3 Are the transcription activators of human ek1 promoter in TSA-Treated human colon carcinoma cells. PLoS One 11: e0147886, 2016.

20. Walter P and Ron D: The unfolded protein response: From stress pathway to homeostatic regulation. Science 334: 1081-1086, 2011.

21. Wang M and Kaufman RJ: The impact of the endoplasmic reticulum protein-folding environment on cancer development. Nat Rev Cancer 14: 581-597, 2014.

22. Namba T, Chu K, Kodama R, Byun S, Yoon KW, Hiraki M, Mandinova A and Lee SW: Loss of p53 enhances the function of the endoplasmic reticulum through activation of the IRE1 $\alpha / \mathrm{XBP} 1$ pathway. Oncotarget 6: 19990-20001, 2015.

23. He J, Du L, Bao M, Zhang B, Qian H, Zhou Q and Cao Z: Oroxin A inhibits breast cancer cell growth by inducing robust endoplasmic reticulum stress and senescence. Anticancer Drugs 27: 204-215, 2016.

24. Tabas I and Ron D: Integrating the mechanisms of apoptosis induced by endoplasmic reticulum stress. Nat Cell Biol 13: 184-190, 2011.

25. Oyadomari S and Mori M: Roles of CHOP/GADD153 in endoplasmic reticulum stress. Cell Death Differ 11: 381-389, 2004.

26. Levine AJ and Oren M: The first 30 years of p53: Growing ever more complex. Nat Rev Cancer 9: 749-758, 2009.

27. Li J, Lee B and Lee AS: Endoplasmic reticulum stress-induced apoptosis: Multiple pathways and activation of p53-up-regulated modulator of apoptosis (PUMA) and NOXA by p53. J Biol Chem 281: 7260-7270, 2006. 